\title{
Estudio y propuestas para mejorar la gestión de estacionamientos vehiculares en la Escuela Superior Politécnica del Chimborazo.
}

\author{
Study and proposals for improving the vehicle parking management in \\ Chimborazo's Polytechnic Superior School
}

\author{
Fredy Gonzalo Naranjo Silva. ${ }^{1}$, Juan Pablo Palaguachi Sumba. ${ }^{2}$, Carlos Xavier Oleas Lara. ${ }^{3}$ \\ \& José Luis Llamuca Llamuca. ${ }^{4}$
}

\begin{abstract}
.
DOI: https://doi.org/10.33262/cienciadigital.v3i2.457

Since the appearance of automobiles, this one has become the essential mean of transport for today's society, showing during the passing of time an accelerated growth of the vehicle population and consequently the negative effects of this fact, which are evident in cities' environment and public spaces. These problems have been the cause of different studies that have determined strategies to reduce the effects caused by this phenomenon. One of the main strategies used is parking management which deals directly with vehicle organization. This research analyses the problem of parking spaces on Chimborazo's Polytechnic Superior School campus from Ecuador considering its environment, facilities, conflictive areas, and parking demand in order to provide solutions that improve mobility and external appearance inside the campus.
\end{abstract}

Keywords: Mobility, Management, Parking.

${ }^{1}$ Escuela Superior Politécnica del Chimborazo, Instituto de Posgrado y Educación Continua, Riobamba, Ecuador, freddynar71@gmail.com

${ }^{2}$ Escuela Superior Politécnica del Chimborazo, Facultad de Administración de Empresas, Carrera de Gestión del Transporte, Riobamba, Ecuador, juan.palaguachi@espoch.edu.ec

${ }^{3}$ Escuela Superior Politécnica del Chimborazo, Facultad de Administración de Empresas, Carrera de Gestión del Transporte, Riobamba, Ecuador, carlos.oleas@espoch.edu.ec

${ }^{4}$ Universidad Escuela Superior Politécnica del Chimborazo, Facultad de Administración de Empresas, Carrera de Gestión del Transporte, Riobamba, Ecuador, jose.llamuca@espoch.edu.ec 


\section{Resumen}

Desde la aparición del automóvil este se ha convertido en el medio transporte imprescindible para la sociedad actual, mostrando con el pasar del tiempo un acelerado crecimiento de la población vehicular y por consiguiente los efectos negativos que este medio transporte provocan en el medio ambiente, el entorno de las ciudades y espacios públicos con alta afluencia de personas. Estos problemas han sido motivo de diferentes estudios que han determinado estrategias para reducir los efectos que provoca este fenómeno. Una de las principales estrategias utilizada es la gestión de estacionamientos la cual resulta imprescindible por su relación directa con este medio de transporte. La presente investigación muestra la problemática del estacionamiento en el campus de la Escuela Superior Politécnica de Chimborazo en Ecuador analizando el entorno, las instalaciones que posee, las zonas más conflictivas, la demanda de estacionamiento, para dar soluciones que mejoren la movilidad e imagen al interior del campus.

Palabras claves: Movilidad, Gestión, Estacionamiento

\section{Introducción.}

El medio de transporte en las ciudades de Latinoamérica se ha ido convirtiendo con el paso del tiempo en un problema de grandes proporciones debido fundamentalmente a la dependencia que el uso del vehículo privado ha creado en la población. Como consecuencia de esta dependencia se ha producido un enorme crecimiento en la utilización del vehículo particular en las ciudades en vías de desarrollo ocasionando el agravamiento de problemas como congestión, contaminación, ocupación vehicular (CEPAL \& GTZ, 2003). A este problema se suman la falta de políticas y planificación que contribuyan a mejorar las condiciones de movilidad y permitan un desarrollo sostenible de las áreas urbanas en las que la población realiza sus actividades cotidianas (Franco, 2014). Los campus universitarios por el tamaño de la población, las grandes extensiones que generalmente presentan, la concentración de actividades en su interior, se consideran micro ciudades lo que conlleva a que presenten los mismos problemas de las ciudades, observando al interior de sus campus grandes flujos vehicular e intensa actividad peatonal ( Urban Land Institute \& National Parking Association, 2014).

En el Ecuador, en la ciudad de Riobamba no ha permanecido indiferente ante esta realidad observando un incremento acelerado del parque automotor, situación que genera los problemas ya conocidos de este fenómeno. La Escuela Superior Politécnica de Chimborazo es uno de los principales y más grandes instituciones de educación superior en el Ecuador, la cual se encuentra ubicado en la parte periférica de Riobamba, cuenta con una población universitaria de alrededor de 16000 personas distribuida de la siguiente manera: 1170 docentes, 590 directivos, personal administrativo y trabajadores y 14240 estudiantes. Esta 
universidad objetivo de la presente investigación ha visto como los efectos del crecimiento de la dependencia del automóvil como medio de transporte habitual se ha ido tomando el interior del campus, observándose una descontrolada ocupación vehicular a lo largo de todas sus instalaciones, así como el incremento de inseguridad en la movilidad peatonal. En este contexto, ésta investigación pretende estudiar la realidad actual de la ocupación vehicular del campus y analizar posibles soluciones a través de estrategias de gestión de estacionamientos que permitan mejorar la movilidad al interior del campus.

\section{Metodología.}

La investigación se desarrolló por fases. En la primera fase se realizó el levantamiento de información primaria, mediante la aplicación de encuestas y formularios elaborados para este fin, las cuales permitieron obtener datos relacionados a la movilidad de las personas dentro de la ESPOCH, conteos vehiculares, horarios de ingreso y salida de vehículos; y, oferta - demanda de estacionamientos.

Para calcular el número de encuestas, para una población total de 16000 personas, se aplicó la fórmula para determinar el tamaño de la muestra para poblaciones finitas (Ec. 1), obteniendo un resultado de 375 encuestas que debieron ser levantadas.

$$
n=\frac{N * Z^{2} * p * q}{e^{2}(N-1)+\left(Z^{2} * p * q\right)}
$$

Dónde:

- $\mathrm{n}=$ Tamaño de la muestra

- $\mathrm{N}=$ Población o universo

- $\mathrm{Z}=$ Nivel de confianza, 1- $\alpha$

- $\mathrm{p}=$ Probabilidad a favor, 0.50

- $\mathrm{q}=$ Probabilidad en contra, 1-0.50

- $\mathrm{e}=$ error muestral, 0.05

Luego de conocer que se requieren levantar 375 encuestas, se procedió a distribuirlas de acuerdo a cada población (Tabla 1).

Tabla 1. Distribución del número de encuestas levantadas en función de la población.

\begin{tabular}{lcc}
\hline Clasificación & Población & Cantidad de encuestas a levantar \\
\hline Personal administrativo & 368 & 9 \\
Docentes & 1170 & 27 \\
Personal de Servicio & 222 & 5 \\
Estudiantes & 14240 & 334 \\
\hline Total & $\mathbf{1 6 0 0 0}$ & $\mathbf{3 7 5}$ \\
\hline
\end{tabular}

Fuente: Elaboración propia 
Mediante la aplicación de formularios o fichas de pantallas se realizaron conteos vehiculares, para determinar el número de vehículos que circulan en el interior del campus durante día. Para obtener información sobre la cantidad de vehículos que circulan por el campus se tomaron datos durante tres días desde las $06 \mathrm{H} 30$ a las $22 \mathrm{H} 00$ con intervalos de 15 minutos y de acuerdo al tipo de vehículo.

Aplicación de formularios o fichas de pantallas para obtener información de la demanda de estacionamientos dentro de la ESPOCH, la toma de datos se realizó durante 03 días en los horarios de 09H00, $13 \mathrm{H} 00$ y 17H00; para lo cual, se dividió el campus en 15 zonas de acuerdo a la ubicación de facultades y escuelas, para obtener el número de vehículos parqueados en los estacionamientos autorizados y fuera de los mismos.

Paralelamente se realizó el inventario de estacionamientos autorizados dentro de las instalaciones de la ESPOCH para determinar cómo se realiza la gestión actual de estacionamientos en el campus, este proceso se realizó en coordinación con el departamento de Desarrollo Físico y Mantenimiento.

\section{Resultados}

Los datos obtenidos en el levantamiento de información permitieron conocer que el $18.13 \%$ de la población encuestada utiliza vehículo privado para trasladarse, mientras que el modo de transporte más utilizado es el bus con un 40,80\%, seguido del transporte a pie con un 27,47\% (Gráfico 1).

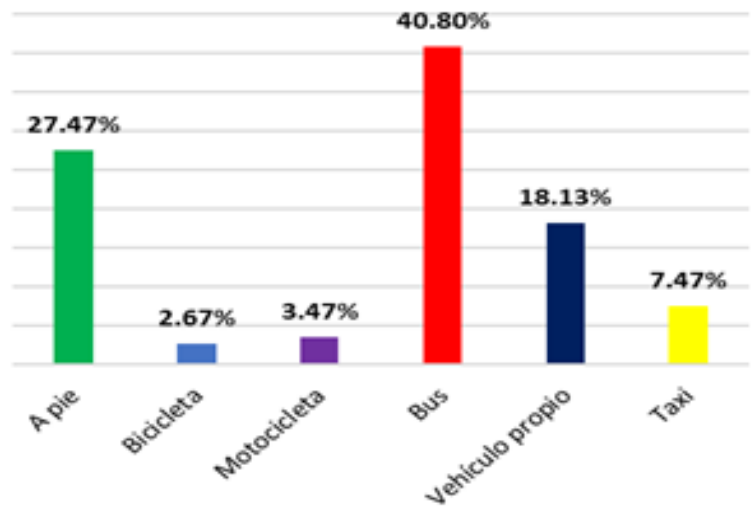

Gráfico 1. Modos de transporte utilizado para movilizarse dentro de la ESPOCH. Fuente: Elaboración propia.

\section{Conteo vehicular}

Los conteos vehiculares permitieron conocer que dentro del campus de la ESPOCH circulan diariamente alrededor de 12548 vehículos que ingresan por los tres accesos habilitados, de los cuales el $56,52 \%$ corresponden a vehículos privados, el 36,40\% son taxis y únicamente el 1,69\% está relacionado con la bicicleta (Tabla 2). 
Tabla 2. Cantidad de vehículos que circulan diariamente dentro de la ESPOCH.

\begin{tabular}{ccccccccc}
\hline Ingreso & \multicolumn{8}{c}{ Tipo de Vehículo } \\
\cline { 2 - 9 } ESPOCH & Particular & Taxi & Furgoneta & Bus & Bicicleta & Moto & Otro & Total \\
\hline 1 & 2164 & 1653 & 25 & 38 & 61 & 131 & 4 & 4076 \\
2 & 2310 & 1201 & 26 & 48 & 64 & 155 & 13 & 3817 \\
3 & 2618 & 1717 & 40 & 44 & 87 & 138 & 11 & 4655 \\
\hline Subtotal & $\mathbf{7 0 9 2}$ & $\mathbf{4 5 7 1}$ & $\mathbf{9 1}$ & $\mathbf{1 3 0}$ & $\mathbf{2 1 2}$ & $\mathbf{4 2 4}$ & $\mathbf{2 8}$ & $\mathbf{1 2 5 4 8}$ \\
\hline Porcentaje & $\mathbf{5 6 , 5 2 \%}$ & $\mathbf{3 6 , 4 3 \%}$ & $\mathbf{0 , 7 3 \%}$ & $\mathbf{1 , 0 4 \%}$ & $\mathbf{1 , 6 9 \%}$ & $\mathbf{3 , 3 8 \%}$ & $\mathbf{0 , 2 2 \%}$ & $\mathbf{1 0 0 \%}$ \\
\hline
\end{tabular}

Fuente: Elaboración propia

Esto hace ver que no existen políticas ni estrategias para controlar el uso indiscriminado del vehículo privado y que tampoco existen políticas para incentivar el uso de modos de transporte sostenible como la bicicleta.

El horario de ingreso a la ESPOCH en el que se registra la mayor afluencia, es entre las 07:00 y 09:30, con un 88,53\% del total del parque automotor que circulan dentro de las instalaciones (Gráfico 2), mientras que el horario en el que salen la mayor cantidad de vehículos se da entre las 11:30 y 13:30 con un 38,40\% (Gráfico 3).

$88.53 \%$

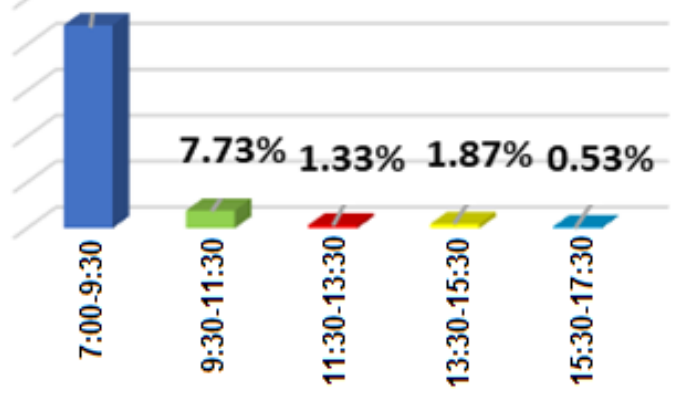

Gráfico 2. Horario de ingreso de vehículos. Fuente: Elaboración propia.
$38.40 \%$

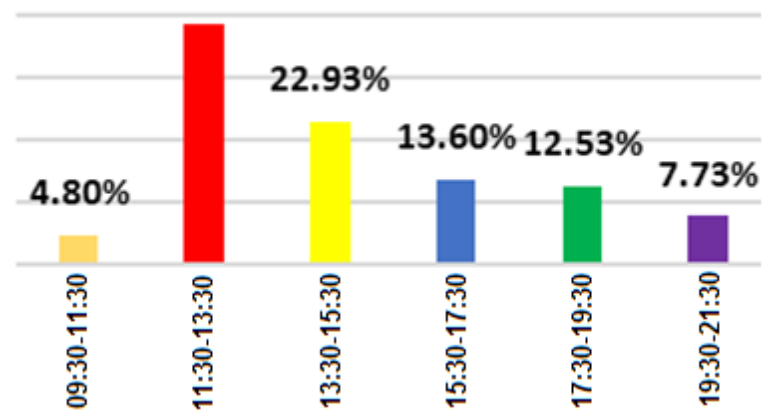

Gráfico 3. Horario de salida de vehículos.

Fuente: Elaboración propia.

De la población encuestada que se moviliza en vehiculó particular, el 39\% no obtiene estacionamiento con facilidad mientras el $61 \%$ si lo obtiene, pero no se especifica la zona de estacionamiento (Gráfico 4).

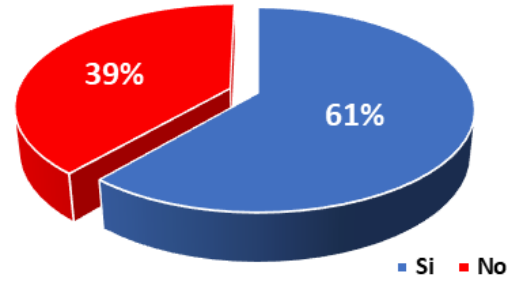

Gráfico 4. Facilidad de encontrar estacionamiento en la ESPOCH.

Fuente: Elaboración propia. 


\section{Oferta de estacionamientos.}

El levantamiento de información en las 15 zonas definidas para el estudio permitió conocer la oferta actual de estacionamientos con los que cuenta la ESPOCH (Tabla 3).

Tabla 3. Oferta de estacionamientos por zona dentro del área de la ESPOCH.

Oferta de estacionamientos por zona dentro de la ESPOCH

\begin{tabular}{|c|c|c|c|c|c|c|c|}
\hline \multirow{2}{*}{ Zona } & \multirow{2}{*}{ Bloque } & \multirow{2}{*}{ Oferta } & \multirow{2}{*}{ Porcentaje } & \multicolumn{2}{|c|}{ Dimensión (m) } & \multirow{2}{*}{$\begin{array}{c}\text { Cumple } \\
\text { norma } \\
\text { INEN }\end{array}$} & \multirow{2}{*}{$\begin{array}{c}\text { Plaza para } \\
\text { discapacitados }\end{array}$} \\
\hline & & & & Largo & Acho & & \\
\hline 1 & Finanzas / Transporte & 55 & $10 \%$ & 2,50 & 5,00 & $\mathrm{Si}$ & No \\
\hline 2 & FADE / Nutrición & 64 & $11 \%$ & 2,50 & 5,00 & $\mathrm{Si}$ & No \\
\hline 3 & Mecánica & 17 & $3 \%$ & 4,50 & 5,00 & No & No \\
\hline 4 & Estadio & 99 & $18 \%$ & 2,50 & 5,00 & $\mathrm{Si}$ & No \\
\hline 5 & Coliseo & 36 & $6 \%$ & 2,50 & 5,00 & $\mathrm{Si}$ & No \\
\hline 6 & Piscina & 46 & $8 \%$ & 2,50 & 5,00 & $\mathrm{Si}$ & No \\
\hline 7 & Edificio Central & 40 & $7 \%$ & 2,50 & 5,00 & $\mathrm{Si}$ & No \\
\hline 8 & FIE / Medicina / Idiomas & 26 & $5 \%$ & 2,50 & 5,00 & $\mathrm{Si}$ & No \\
\hline 9 & IPEC / Ciencias & 21 & $4 \%$ & 2,50 & 5,00 & $\mathrm{Si}$ & No \\
\hline 10 & Sistemas & 47 & $8 \%$ & 2,50 & 5,00 & $\mathrm{Si}$ & No \\
\hline 11 & Auditorio de Ciencia Pecuarias & 20 & $4 \%$ & 2,70 & 5,00 & No & No \\
\hline 12 & Zootecnia & 25 & $4 \%$ & 2,60 & 5,00 & No & No \\
\hline 13 & Unidad Investigaciones Porcinas & 17 & $3 \%$ & 2,50 & 5,00 & $\mathrm{Si}$ & No \\
\hline 14 & Ecoturismo & 26 & $5 \%$ & 2,50 & 5,00 & $\mathrm{Si}$ & No \\
\hline 15 & Agronomía & 18 & $3 \%$ & 2,50 & 5,00 & $\mathrm{Si}$ & No \\
\hline
\end{tabular}

Fuente: Elaboración propia

La oferta total de estacionamientos dentro de la ESPOCH es de 557 plazas, de la cuales el porcentaje más alto está ubicado en la zona 4 con el $18 \%$ y el más bajo en un $3 \%$ se encuentra en las zonas 3,13 y 15 .

\section{Demanda de estacionamientos.}

El conteo vehicular en las 15 zonas dentro de la ESPOCH, permitió conocer los datos relacionados a la demanda actual de estacionamientos, en el horario de las 09:00, 13:00, 17:00 (Tabla 4).

Tabla 4. Demanda de estacionamientos en el área de la ESPOCH.

\begin{tabular}{clcccc}
\hline \multicolumn{5}{c}{ Demanda de estacionamientos en el área de la ESPOCH } \\
\hline \multirow{2}{*}{ Zona } & \multirow{2}{*}{ Bloque } & \multicolumn{2}{c}{ Número de vehículos por horario } & \multirow{2}{*}{ Promedio } \\
\cline { 3 - 5 } & & $\mathbf{9 : 0 0}$ & $\mathbf{1 3 : 0 0}$ & $\mathbf{1 7 : 0 0}$ & \\
\hline 1 & Finanzas / Transporte & 55 & 32 & 33 & 40 \\
2 & FADE / Nutrición & 201 & 93 & 133 & 142 \\
3 & Mecánica & 114 & 24 & 60 & 66 \\
4 & Estadio & 20 & 34 & 35 & 30 \\
5 & Coliseo & 3 & 9 & 12 & 8 \\
6 & Piscina & 1 & 3 & 6 & 3
\end{tabular}




\begin{tabular}{clcccc}
\hline 7 & Edificio Central & 71 & 60 & 77 & 69 \\
8 & FIE / Medicina / Idiomas & 147 & 76 & 69 & 97 \\
9 & IPEC / Ciencias & 148 & 103 & 142 & 131 \\
10 & Sistemas & 74 & 38 & 77 & 63 \\
11 & Auditorio de Ciencia Pecuarias & 3 & 4 & 3 & 3 \\
12 & Zootecnia & 43 & 25 & 26 & 31 \\
13 & Unidad de Investigaciones Porcinas & 12 & 0 & 0 & 4 \\
14 & Ecoturismo & 2 & 9 & 8 & 6 \\
15 & Agronomía & 48 & 19 & 14 & 27 \\
\hline & & $\mathbf{9 4 2}$ & $\mathbf{5 2 9}$ & $\mathbf{6 9 5}$ & $\mathbf{7 2 2}$ \\
\hline
\end{tabular}

Fuente: Elaboración propia.

De acuerdo a estos datos, se observa que existe un déficit de estacionamientos en las zonas $2-3,7-10,12$ y 15, lo que genera un alto índice vehículos estacionados en lugares prohibidos (ver Figura 7).

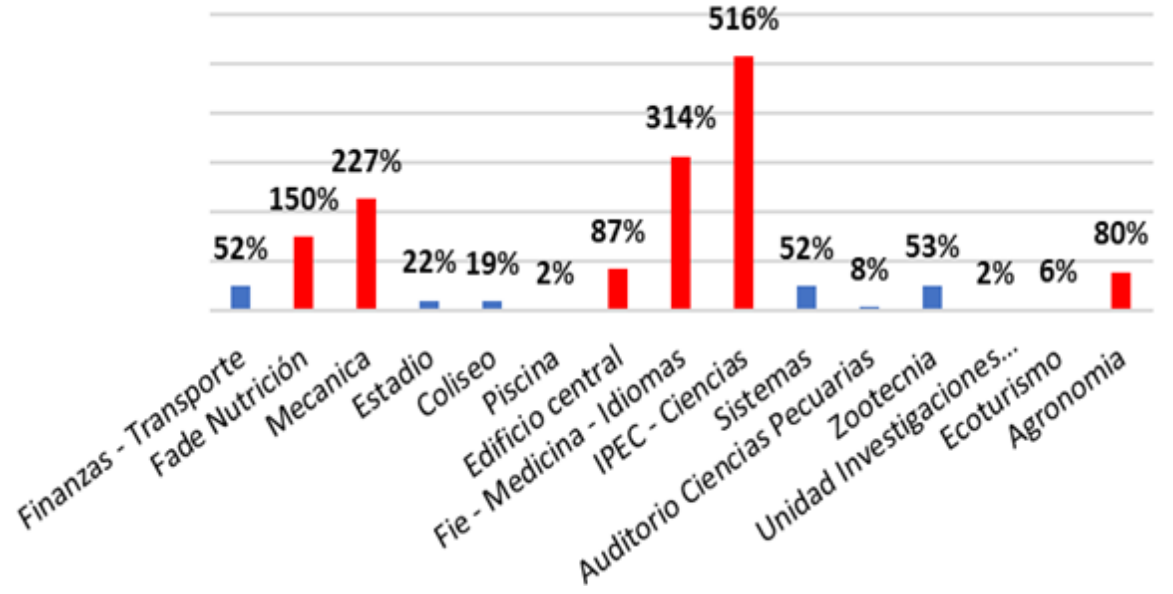

Gráfico 5. Porcentaje de ocupación de estacionamientos en la ESPOCH.

Fuente: Elaboración propia.

Por otro lado, mediante la aplicación de la normativa para usos de suelo obtenida del Distrito Metropolitano de Quito (Concejo Metropolitano de Quito, 2012); que es, la que más se acopla a la realidad de la ESPOCH, se realizó el dimensionamiento de demanda de estacionamientos en función del inventario existente en las 15 zonas (Tabla 5).

Tabla 5. Dimensionamiento de demanda de estacionamientos según la Normativa para usos de suelo. Dimensionamiento de demanda de estacionamientos por zonas para la ESPOCH

\begin{tabular}{clcc}
\hline No. & \multicolumn{1}{c}{ Bloque } & Cantidad de vehículos & Porcentaje \\
\hline 1 & Finanzas / Transporte & 136 & $6 \%$ \\
2 & FADE / Nutrición & 288 & $12 \%$ \\
3 & Mecánica & 179 & $8 \%$ \\
4 & Estadio & 305 & $13 \%$ \\
5 & Coliseo & 39 & $2 \%$ \\
6 & Piscina & 43 & $2 \%$ \\
7 & Edificio Central & 130 & $5 \%$ \\
8 & FIE / Medicina / Idiomas & 435 & $18 \%$ \\
9 & IPEC / Ciencias & 233 & $10 \%$
\end{tabular}




\begin{tabular}{llcc}
\hline 10 & Sistemas & 68 & $3 \%$ \\
11 & Auditorio de Ciencia Pecuarias & 102 & $4 \%$ \\
12 & Zootecnia & 157 & $7 \%$ \\
13 & Unidad de Investigaciones Porcinas & 43 & $2 \%$ \\
14 & Ecoturismo & 55 & $2 \%$ \\
15 & Agronomía & 159 & $7 \%$ \\
\hline & & $\mathbf{2 3 7 2}$ & $\mathbf{1 0 0 \%}$ \\
\hline
\end{tabular}

Fuente: Elaboración propia.

De acuerdo a la normativa de uso de suelo, se establece una demanda total de 2372 estacionamientos dentro de la ESPOCH; lo cual, si se relaciona con la oferta actual de 557 espacios, se determinó una demanda insatisfecha de 1814 plazas de parqueo.

\section{Propuesta}

El diagnóstico de la situación actual permitió conocer que en la ESPOCH existe un déficit en cuanto el número de plazas de parqueo; por lo que, se plantean medidas de gestión de estacionamientos que busca satisfacer la demanda actual, que a su vez ayudaran a mejorar la movilidad en el interior del campus.

\section{Readecuación de plazas de estacionamiento de acuerdo a la norma técnica RTE INEN 004 - 2:2011.}

El estudio determinó que la infraestructura actual de estacionamientos que disponen las zonas 3, 11 y 12, no están diseñadas de acuerdo a la norma técnica (Instituto Ecuatoriano de Normalización INEN, 2011); por lo que, es necesario la readecuación de estas plazas de parqueo, considerando además que deben existir estacionamientos exclusivos para discapacitados. En este contexto, en la (Tabla 6) se presenta el cálculo del número de plazas de estacionamientos aplicando la norma técnica, en las zonas 3, 11 y 12, lo cual permitió visualizar un incremento en la oferta, de 53 sitios de parqueo normal y 26 plazas para discapacitados, mejorando su distribución y optimizando el espacio físico en las mencionadas zonas.

Tabla 6. Plazas de estacionamientos calculados según la norma técnica RTE INEN 004 - 2:2011.

\begin{tabular}{clccc}
\hline Zona & Bloque & Oferta & $\begin{array}{c}\text { No. plazas según } \\
\text { norma INEN }\end{array}$ & $\begin{array}{c}\text { No. plazas para } \\
\text { discapacitados }\end{array}$ \\
\hline 1 & Finanzas / Transporte & 55 & 0 & 2 \\
2 & FADE / Nutrición & 64 & 0 & 3 \\
3 & Mecánica & 17 & 40 & 2 \\
4 & Estadio & 99 & 0 & 4 \\
5 & Coliseo & 36 & 0 & 1 \\
6 & Piscina & 46 & 0 & 2 \\
7 & Edificio Central & 40 & 0 & 2 \\
8 & FIE / Medicina / Idiomas & 26 & 0 & 1 \\
9 & IPEC / Ciencias & 21 & 0 & 1
\end{tabular}




\begin{tabular}{llccc}
\hline 10 & Sistemas & 47 & 0 & 2 \\
11 & Auditorio de Ciencia Pecuarias & 20 & 40 & 2 \\
12 & Zootecnia & 25 & 35 & 1 \\
13 & Unidad de Investigaciones Porcinas & 17 & 0 & 1 \\
14 & Ecoturismo & 26 & 0 & 1 \\
15 & Agronomía & 18 & 0 & 1 \\
\hline & Total & $\mathbf{5 5 7}$ & $\mathbf{1 1 5}$ & $\mathbf{2 6}$ \\
\hline
\end{tabular}

Fuente: Elaboración propia.

Solo tomando en cuenta esta medida se logrará incrementar en aproximadamente un 9\%, la oferta de estacionamientos, dotando al campus de un total de 610 plazas de parqueo.

\section{Creación de nuevas plazas de estacionamiento.}

La propuesta de creación de nuevas plazas de estacionamiento, está enfocado a satisfacer la demanda en las zonas que presenta un alto déficit de espacios de parqueo. Para el cálculo del número de celdas necesarias para cubrir dicha necesidad, se consideró el promedio entre la demanda por uso de suelo y el número de vehículos estacionados en una hora pico; y, al resultado obtenido con esta relación se aplicó el percentil 85, que según algunos autores consideran el recomendado para establecer la capacidad efectiva (Secretaría de Territorio de la Alcaldía de Quito, 2015), dando como resultado los valores de la (Tabla 8).

Tabla 7. Número de plazas de estacionamiento calculadas para zonas con alto déficit.

\begin{tabular}{clccc} 
Zona & Bloque & $\begin{array}{c}\text { No. plazas para } \\
\text { discapacitados }\end{array}$ & $\begin{array}{c}\text { No. plazas } \\
\text { calculadas }\end{array}$ & $\begin{array}{c}\text { Total de plazas } \\
\text { requeridas }\end{array}$ \\
\hline 2 & FADE / Nutrición & 5 & 125 & 130 \\
3 & Mecánica & 3 & 84 & 87 \\
7 & Edificio Central & 2 & 50 & 52 \\
8 & FIE / Medicina / Idiomas & 8 & 204 & 212 \\
9 & IPEC / Ciencias & 5 & 133 & 138 \\
10 & Sistemas & 1 & 74 & 75 \\
12 & Zootecnia & 2 & 59 & 61 \\
15 & Agronomía & 3 & 64 & 67 \\
\hline
\end{tabular}

Fuente: Elaboración propia.

El cálculo teórico determinó que se requieren alrededor de 822 plazas para satisfacer la demanda en las zonas que presentan un alto déficit de sitios de estacionamiento. Por ello, se ha delimitado un área (Figura 1) para la ubicación de la nueva oferta de celdas de parqueo, considerando una distancia máxima de $400 \mathrm{~m}$ de recorrido para el peatón, entre el estacionamiento y las zonas intervenidas. 


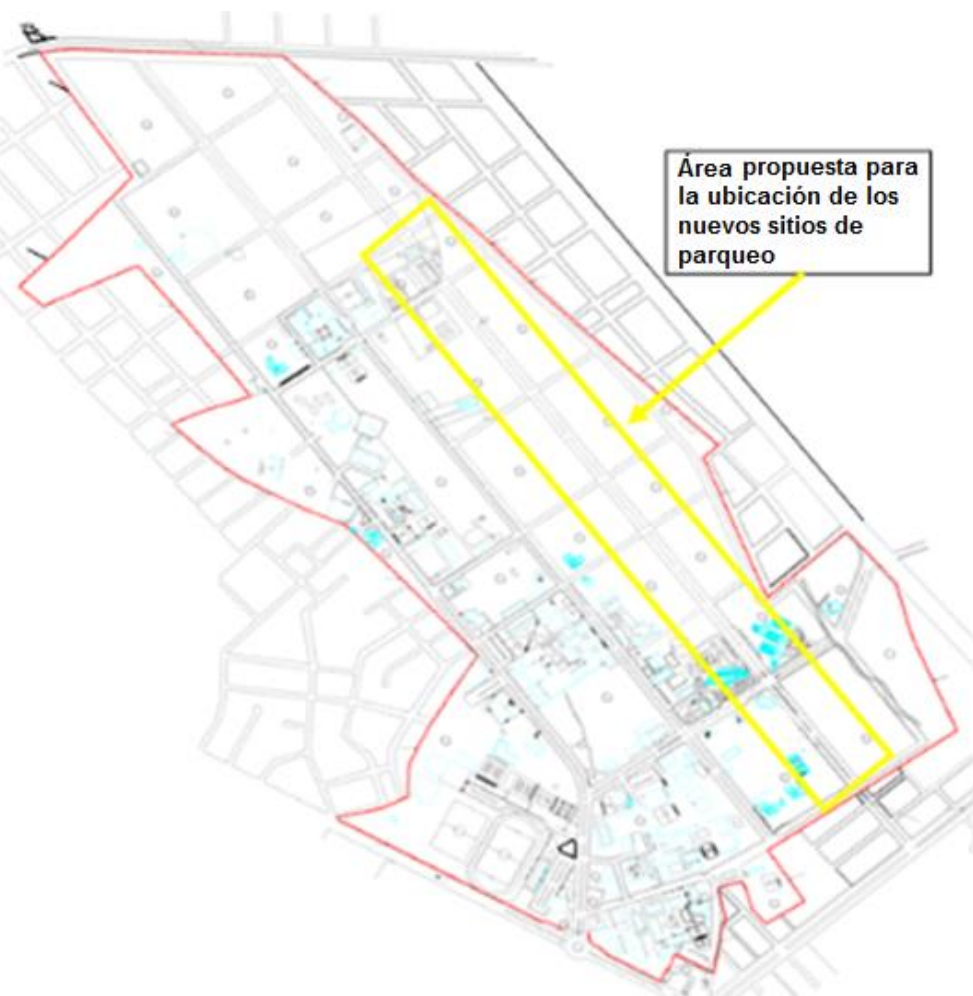

Figura 1. Área delimitada para la ubicación de la nueva oferta de estacionamientos.

Fuente: Elaboración propia.

\section{Establecer un modelo de gestión para la administración del tránsito y movilidad en la ESPOCH.}

Actualmente, la ESPOCH no cuenta con un modelo de gestión para la administración del tránsito y la movilidad en el interior de su campus; por lo que, se propone realizar un estudio que permita definir el modelo de gestión adecuado; y en función de este, crear un departamento que se encargue de proponer políticas, así como de la planificación, regulación y control del tránsito y movilidad en el interior del campus.

Entre la primeras acciones que deberá realizar el departamento de tránsito y movilidad será implementar los estudios realizados; así como, definir áreas específicas de estacionamiento para autoridades, administrativos, docentes, estudiantes e invitados, los cuales dispondrán de permisos según sea el caso, para la correcta utilización de todos estos espacios con el fin de mejorar el ordenamiento vehicular dentro de la ESPOCH.

\section{Construcción de accesos directos desde el exterior hacia las áreas de parqueo internas de la ESPOCH.}

Como estrategia complementaria a la creación de nuevos estacionamientos, se propone la construcción de vías de acceso directo desde el exterior de la ESPOCH hacia los estacionamientos ubicados en el interior del campus, partiendo desde la avenida Milton 
Reyes hasta su salida por la avenida Leónidas Proaño (Figura 2), con esta acción se pretende reducir el flujo vehicular en el interior del campus, generado por la búsqueda de un lugar para estacionarse.

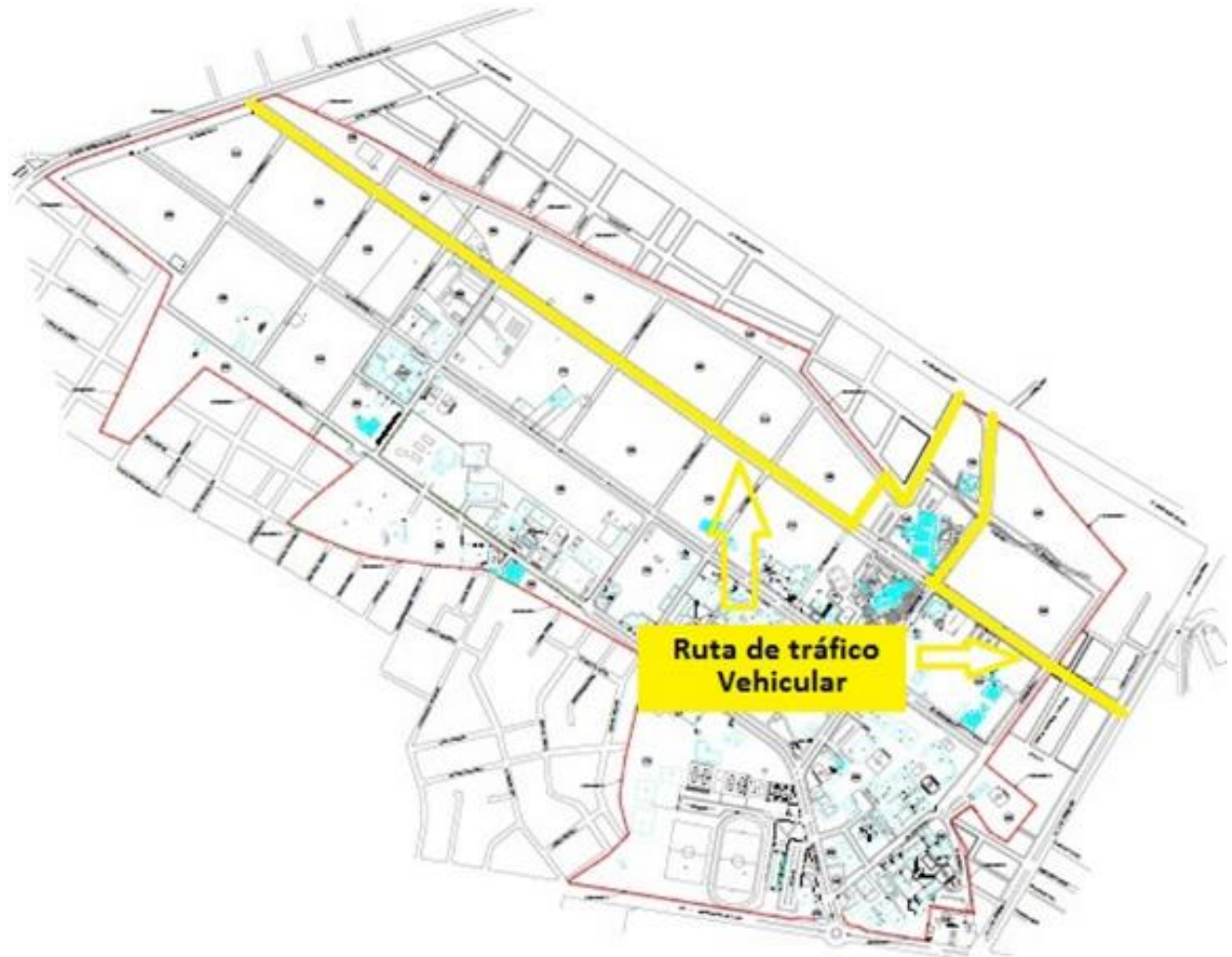

Figura 2. Propuesta de accesos directos a la nueva área de estacionamientos.

Fuente: Elaboración propia.

\section{Conclusiones.}

- La investigación permitió determinar que existe un alto déficit de plazas de estacionamiento en las diferentes zonas dentro de la ESPOCH; por lo que, un gran porcentaje de vehículos se estacionan en sitios prohibidos para este fin sin respetar los lugares de circulación peatonal, paradas de bus y demás espacios públicos.

- El estudio evidenció la inexistencia de un sistema de control vehicular en los accesos de la ESPOCH; así como también, control para el buen uso de las zonas de estacionamiento, esta situación genera problemas en la movilidad y fomenta la libre ocupación de las vías de una manera desordenada.

- La falta de una oferta equilibrada de espacios de estacionamiento en zonas adecuadas para este fin, impide la aplicación de estrategias que contribuyan a un adecuado control de la circulación vehicular al interior del campus. 
- No se cuenta con un departamento de movilidad que se encargue de planificación, regulación y control de tránsito y movilidad en las instalaciones internas de la ESPOCH.

\section{Referencias bibliográficas.}

Urban Land Institute, \& National Parking Association. (2014). The Dimensions of Parking by Urban Land Institute. Washington: Urban Land Institute.

Banco Interamericano de Desarrollo. (2013). Guía práctica: Estacionamiento y políticas de reducción de congestión en América Latina. Obtenido de https://publications.iadb.org/es/publicacion/17409/guia-practica-estacionamiento-ypoliticas-de-reduccion-de-congestion-en-america

CEPAL, \& GTZ. (2003). Congestión del tránsito. El problema y como enfrentarlo. Obtenido de https://repositorio.cepal.org/bitstream/handle/11362/27813/6/S0301049_es.pdf.

Concejo Metropolitano de Quito. (2012). Ordenanza Metropolitana No. O172. Ordenanza Metropolitana que establece el régimen administrativo del suelo en el Distrito Metropolitano de Quito. Obtenido de https://es.scribd.com/document/355780156/Ordenanza-Metropolitana-0172-Quito

Franco, L. (2014). La movilidad sostenible en campus universitarios: una comparación de las mejores prácticas en Estados Unidos y Europa. Aplicabilidad en universidades venezolanas. Obtenido de Revista de la Facultad de Ingeniería Universidad Central de Venezuela, 29(2), 23-40: Recuperado en 05 de mayo de 2019, de http://www.scielo.org.ve/scielo.php?script=sci_arttext\&pid=S0798$40652014000200003 \& \operatorname{lng}=\mathrm{es} \& \ln \mathrm{l}=\mathrm{es}$.

González, C., Moreno , D., \& Velásquez, S. (2011). Análisis de la movilidad en campus universitarios: Caso de estudio Universidad de Antioquia. Obtenido de http://revistas.elpoli.edu.co/index.php/pol/article/view/179/153

Instituto Ecuatoriano de Normalización INEN. (2011). Reglamento técnico Ecuatoriano RTE INEN 004-2:2011. Primera revisión. Señalización Vial. Parte 2. Señalización Horizontal. Obtenido de https://www.obraspublicas.gob.ec: https://www.obraspublicas.gob.ec/wpcontent/uploads/downloads/2015/04/LOTAIP2015_reglamento-tecnicoecuatoriano-rte-inen-004-1-2011.pdf 
Secretaría de Territorio de la Alcaldía de Quito. (2015). Resolución No. STHV-RT-02-2015.

Requisitos Mínimos de Estacionamientos para Vehículos Livianos por Usos. Obtenido de https://www.ecp.ec/wp-content/uploads/2018/01/STHV-RT-02-2015Estacionamientos.pdf 


\section{PARA CITAR EL ARTÍCULO INDEXADO.}

Naranjo Silva, F., Palaguachi Sumba, J., Oleas Lara, C., \& Llamuca Llamuca, J. (2019). Estudio y propuestas para mejorar la gestión de estacionamientos vehiculares en la Escuela Superior Politécnica del Chimborazo. Ciencia Digital, 3(2), 17-30. https://doi.org/10.33262/cienciadigital.v3i2.457

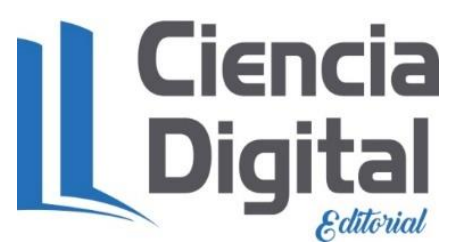

El artículo que se publica es de exclusiva responsabilidad de los autores y no necesariamente reflejan el pensamiento de la Revista Ciencia Digital.

El artículo queda en propiedad de la revista y, por tanto, su publicación parcial y/o total en otro medio tiene que ser autorizado por el director de la Revista Ciencia Digital.
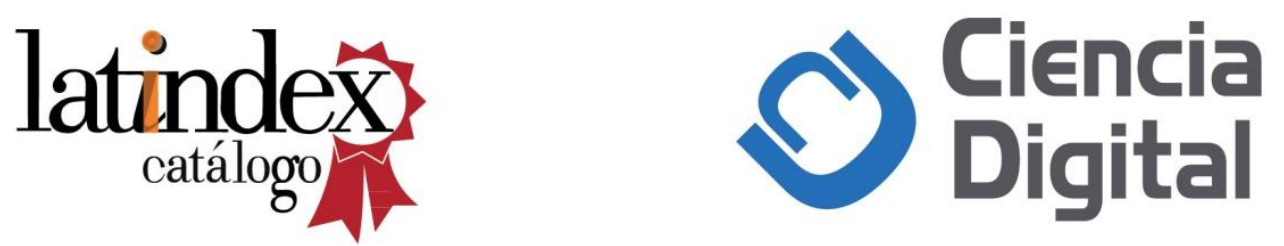